\title{
ENFERMAGEM E O MANEJO DOS RESÍDUOS DE SERVIÇOS DE SAÚDE: UMA REVISÃO INTEGRATIVA
}

\author{
NURSING AND THE MANAGEMENT OF HEALTH SERVICES WASTE: AN \\ INTEGRATIVE REVIEW
}

Luiz Fernando Brito da Costa ${ }^{1} *$ Francisca Adriana Barreto $^{2}$

\section{RESUMO}

Objetivo: conhecer a contribuição da enfermagem para a sustentabilidade ambiental com foco nos resíduos de serviços de saúde. Método: estudo do tipo revisão integrativa da literatura, com busca na base de dados Google Acadêmico, abrangendo estudos no período entre 2018 a 2021. Resultados: evidenciam-se os problemas relacionados à falta do conhecimento acerca dos resíduos de serviços de saúde que acarreta em efeitos sob a saúde humana e ambiental. Conclusão: o manejo inadequado ofertado pela enfermagem afeta o meio ambiente, haja vista que o conhecimento acerca do gerenciamento se torna fundamental para a redução dos efeitos no meio natural.

Palavras-chave: Sustentabilidade; Enfermagem; Resíduos de Serviços de Saúde; Meio Ambiente.

\section{ABSTRACT}

Objective: to know the contribution of nursing to environmental sustainability with a focus on waste from health services. Method: an integrative literature review study, with a search in the Google Academic database, covering studies from 2018 to 2021. Results: the problems related to the lack of knowledge about the waste of health services are evidenced. under human and environmental health. Conclusion: the inadequate management offered by nursing affects the environment, given that knowledge about management becomes essential to reduce the effects on the natural environment.

Keywords: Sustainability; Nursing; Health Services Waste; Environment.

\footnotetext{
${ }^{1}$ Graduando em enfermagem pela Universidade do Estado do Rio Grande do Norte, Pau dos Ferros/RN - Brasil. ORCID: https://orcid.org/0000-0002-8868-0850.

${ }^{2}$ Enfermeira; Doutora em Geografia; Professora Adjunto IV da Universidade do Estado do Rio Grande do Norte, Pau dos Ferros/RN

- Brasil. ORCID: https://orcid.org/0000-0002-5183-043X.
} 


\section{INTRODUÇÃO}

A Lei $\mathrm{n}^{\circ} 8.080 / 90$ estabelece que a saúde é um direito do ser humano e dever do Estado, que deve garantir políticas públicas que visem a redução de riscos e agravos à saúde, e o meio ambiente é um dos fatores determinantes e condicionantes para $o$ processo de adoecimento ${ }^{(1)}$. Desse modo, a saúde ambiental é resultante da interação entre a sociedade e a natureza, partindo da geração de resíduos nos quais podem afetar diretamente o processo saúde-doença e a qualidade de vida dos seres vivos ${ }^{(2)}$.

No Brasil, são gerados cerca de 79 milhões de toneladas de resíduos sólidos urbanos (RSU), e parte destes são compostos pelos resíduos dos serviços de saúde (RSS), e quando seu manejo acontece de forma inadequada caracteriza riscos à saúde humana e ambiental $^{(3)}$. Os RSS são oriundos da prestação da assistência à saúde, desde instituições públicas ou privadas, domicílios à estabelecimentos que manuseiam esses tipos de resíduos produzidos durante $\mathrm{o}$ atendimento $^{(4)}$.

Os resíduos de serviços de saúde são classificados em cinco grupos: grupo A infectantes; Grupo B - químicos; Grupo C radioativos; Grupo D - resíduos comuns; Grupo E - perfuro cortantes ${ }^{(5)}$. E apresentam fatores determinantes a exposição de agentes infecciosos, químicos, radioativos e até mesmo objetos perfurocortantes, no que pode levar a transmissão de doenças infecto- contagiantes por vírus e bactérias, como por exemplo, o vírus da imunodeficiência humana (HIV), os vírus da hepatite $\mathrm{B}$ e $\mathrm{C}$, entre outros $^{(6)}$. Quando os resíduos produzidos não são gerenciados conforme a adequação, pode acarretar efeitos adversos a saúde populacional e ao seu meio, uma vez que, leva a contaminação das características ambientais como água, solo e ar ${ }^{(4)}$.

Para que o gerenciamento seja realizado pelos serviços de saúde, se faz necessário lançar mão da implementação de políticas públicas que desenvolvam ações que estimulem a educação, a consciência e a responsabilidade social para com o comprometimento pelo meio ambiente. Nisto, objetiva-se a minimização dos impactos à saúde ambiental ocasionado pelo descarte incorreto dos resíduos ${ }^{(4)}$. Com isso, é imprescindível que haja a promoção da saúde e preservação do meio ambiente. Assim, cabe aos gestores das esferas municipais, estaduais e federais adotarem na prática o funcionamento das legislações e normatizações responsáveis pelo ambiente natural $^{(3)}$.

Para tanto, todo estabelecimento proveniente da saúde deve contar com a elaboração de um plano de gerenciamento correto dos resíduos dos serviços de saúde (PGRSS), no qual corresponde a compatibilidade de normas relacionadas ao manejo adequado, desde segregação a disposição final $^{(5)}$. Devido a alta 
periculosidade de alguns resíduos, que não possuem uma degradação de forma natural, se deve estimular a conscientização para um ambiente sustentável, corroborando com as legislações na perspectiva de reduzir a ação prejudicial ao meio natural relacionado ao descarte incorreto dos $\operatorname{lixos}^{(7)}$.

As instituições de saúde promovem a exposição de riscos à saúde humana $\mathrm{e}$ consequentemente ao meio natural. Os enfermeiros exercem da sensibilidade para as necessidades vulneráveis, tendo papel fundamental no compartilhamento de seus conhecimentos para a promoção da saúde, tanto humana quanto ambiental, já que há comprometimento com a qualidade de vida dos indivíduos como um todo ${ }^{(8)}$.

Os problemas ambientais estão correlacionados com a evolução do desenvolvimento econômico e social ao longo dos tempos, acarretando em questões ambientalistas que afetam a saúde. Tanto, para que a saúde ambiental se mantenha preservada é necessário a tomada de ações que reflitam positivamente no ecossistema, como políticas de proteção e promoção a saúde ambiental e humana. Contudo, é imprescindível que haja a participação da enfermagem na elaboração de estratégias de cuidado para educar e conscientizar a população, para que mantenham atitudes sustentáveis com o meio ambiente, uma vez que, a enfermagem tem capacidade de influenciar pessoas por meio da educação em saúde $^{(9)}$.

Portanto, faz-se mister salientar a importância do conhecimento relacionado ao gerenciamento adequado dos RSS pelos profissionais da enfermagem, bem como, a sustentabilidade do meio ambiente. Para isto, a questão levantada para o desenvolvimento dessa revisão, foi: Quais as contribuições da enfermagem para a sustentabilidade acerca dos RSS? Mediante isso, apresenta como objetivo conhecer a contribuição da enfermagem para a sustentabilidade ambiental com foco nos resíduos de serviços de saúde.

\section{MÉTODO}

Trata-se de um estudo bibliográfico, do tipo revisão integrativa da literatura, um método de pesquisa que permite a sintetização de evidências clínicas de forma sistemática, de modo a incorporar os resultados científicos $^{(10)}$. Os dados foram coletados no mês de junho de 2021, na base de dados do Google Acadêmico, pois a pesquisa prévia na Biblioteca Virtual em Saúde (BVS) não contemplava o número sub existencial de artigos, abrindo-se preferência pelo Google Acadêmico.

Utilizou-se de descritores como 'Sustentabilidade; Enfermagem; Resíduos de Serviços de Saúde; Meio ambiente", totalizando em 2.420 arquivos. Para a seleção, foram estabelecidos critérios de inclusão: textos completos, últimos quatro anos (2018 - 
2021), título e resumo. Excluíram-se revisões, teses, dissertações, trabalhos publicados em eventos. Após o refinamento dos arquivos, 43 artigos foram selecionados para leitura dos títulos e resumos. Resultou-se no total de 25 artigos para leitura completa. Destes, 18 artigos foram eliminados pós leitura dos textos completos, sete publicações foram escolhidas para o estudo. Conforme mostra a figura 1 - fluxograma PRISMA ${ }^{(11)}$.
Após a análise dos estudos iniciou-se a elaboração do corpo da revisão de forma sintetizada e sistemática a corresponder o objetivo proposto pelo estudo. Assim, é prescindível a discussão posta para a compreensão das questões que envolvem a enfermagem e o meio ambiente, assim como, os riscos expostos ao comprometimento da sustentabilidade ambiental em meio aos resíduos de serviços de saúde dispostos de forma inapropriada.

\section{Figura 1 - Fluxograma PRISMA}

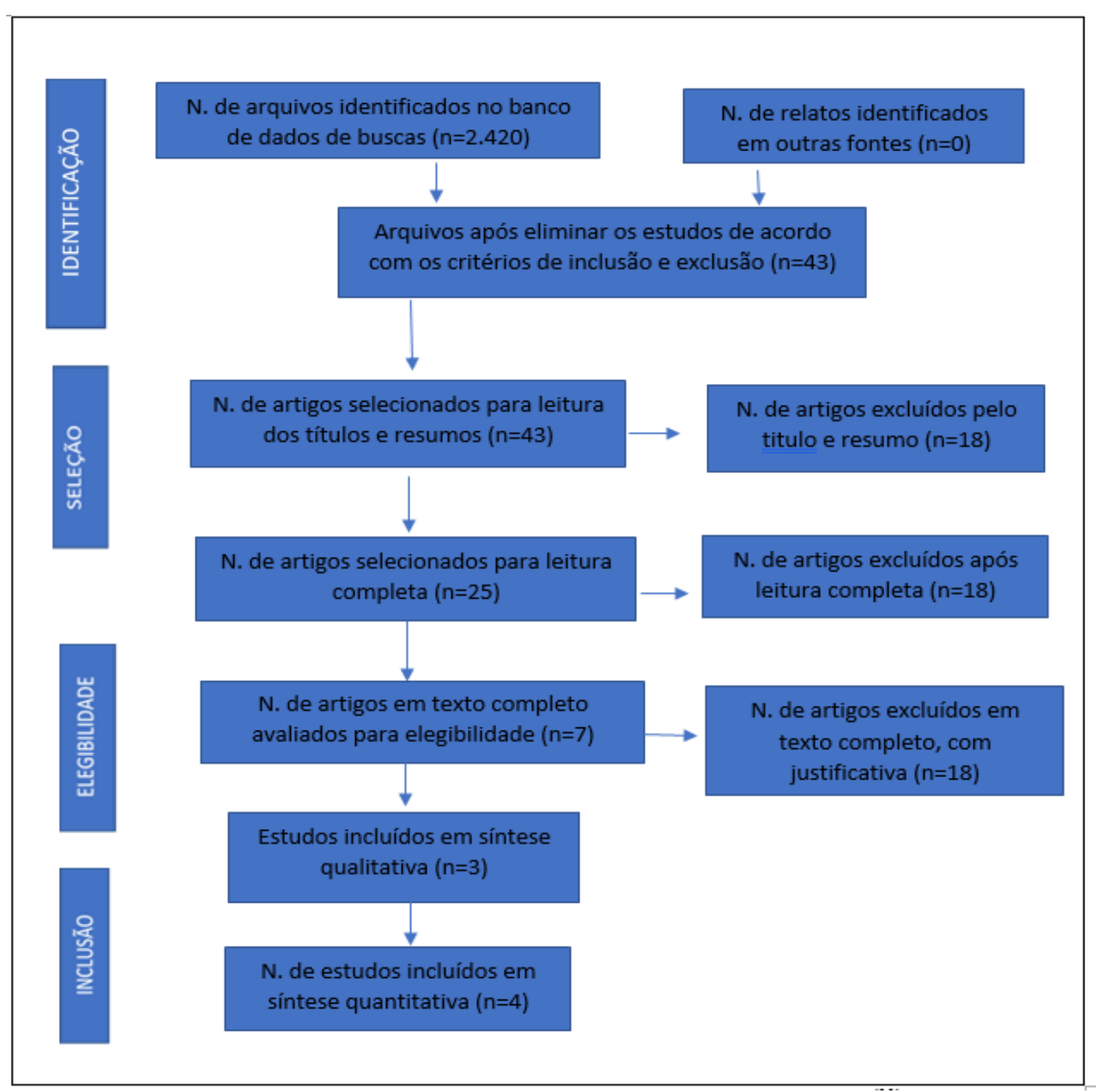

Fonte: Dados da pesquisa, 2021. Baseado na Recomendação PRISMA (2015) ${ }^{(11)}$ 


\section{RESULTADOS}

Quadro 1 - Resultados encontrados

\begin{tabular}{|c|c|c|c|}
\hline TÍTULO/REVISTA/ANO & AUTORES & METODOLOGIA & RESULTADOS \\
\hline $\begin{array}{l}\text { Conhecimento do } \\
\text { enfermeiro acerca do } \\
\text { gerenciamento dos resíduos } \\
\text { de serviços de saúde nas } \\
\text { unidades básicas de saúde } \\
\text { no interior de Goiás, Brasil. } \\
\text { International Journal of } \\
\text { Current Research. 2020. }\end{array}$ & $\begin{array}{l}\text { Rodrigues, A. } \\
\text { F. S; } \\
\text { Meireles, G. } \\
\text { O. A. B; Reis, } \\
\text { M. A; Matos, } \\
\text { M. A; Melo, } \\
\text { L. B; Moura, } \\
\text { S. G; Santos, } \\
\text { N. F. S; } \\
\text { Oliveira, E. } \\
\text { D. F; Moraes, } \\
\text { F. M; Aranha, } \\
\text { T. } \\
\text { Andrade, J. } \\
\text { M; Machado, } \\
\text { D. T. S. }\end{array}$ & $\begin{array}{lr}\text { Estudo descritivo } \\
\text { com abordagem } \\
\text { qualitativa, realizado } \\
\text { em um município em } \\
\text { Goiás, } \\
\text { entrevistados } \\
\text { enfermeiros } \\
\text { atuavam em } \\
\text { Básica dnidade } \\
\text { (UBS). }\end{array}$ & $\begin{array}{l}\text { É perceptível a dificuldade } \\
\text { de compreensão relacionada } \\
\text { ao conhecimento sobre os } \\
\text { RSS, assim como em } \\
\text { classifica-los. }\end{array}$ \\
\hline $\begin{array}{l}\text { Ensino } r \text { sobre } \\
\text { gerenciamento de resíduos } \\
\text { na graduação em } \\
\text { enfermagem: realidade, } \\
\text { reflexões e propostas. } \\
\text { Revista Eletrônica Acervo } \\
\text { Saúde. 2021. }\end{array}$ & $\begin{array}{l}\text { Guimarães, P. } \\
\text { S. S; Vilela, } \\
\text { R. Q. B; } \\
\text { Oliveira, S. } \\
\text { M. B; Silva, } \\
\text { P. J. T. G; } \\
\text { Reis, R. P; } \\
\text { Silva, E. S; } \\
\text { Lima, A. S; } \\
\text { Silva, R. C. }\end{array}$ & $\begin{array}{l}\text { Pesquisa de campo } \\
\text { descritiva e analítica } \\
\text { com abordagem } \\
\text { qualitativa, realizada } \\
\text { por discentes do } \\
\text { curso de } \\
\text { enfermagem, } \\
\text { fazendo-se uso da } \\
\text { técnica grupo focal } \\
\text { (GF). }\end{array}$ & $\begin{array}{l}\text { Os discentes mostraram } \\
\text { conhecimento sobre os RSS, } \\
\text { assim como a abordagem } \\
\text { das questões } \\
\text { biossegurança e o descarte } \\
\text { inadequado, no que pode } \\
\text { acarretar em impactos a } \\
\text { saúde humana e ambiental. } \\
\text { É visto que há } \\
\text { distanciamento entre a teoria } \\
\text { e prática para o manejo } \\
\text { adequado. }\end{array}$ \\
\hline associados & Oliveira, L. P; & analítico & Observou-se \\
\hline
\end{tabular}




\begin{tabular}{|c|c|c|c|}
\hline $\begin{array}{l}\text { manejo adequado de } \\
\text { resíduos de serviços de } \\
\text { saúde entre profissionais de } \\
\text { enfermagem. Rev baiana } \\
\text { enf. 2018. }\end{array}$ & $\begin{array}{l}\text { Mendonça, I. } \\
\text { V. S; Gomes, } \\
\text { S. C. S; } \\
\text { Caldas A. J. } \\
\text { M. }\end{array}$ & $\begin{array}{l}\text { transversal, realizado } \\
\text { em um hospital em } \\
\text { São Luís, Maranhão. } \\
\text { A população deste } \\
\text { estudo foi composta } \\
\text { por } 461 \text { profissionais } \\
\text { de enfermagem. }\end{array}$ & $\begin{array}{l}\text { profissionais } \\
\text { enfermagem demonstraram } \\
\text { conhecimento sobre o } \\
\text { manejo dos RSS, uma vez } \\
\text { que, manuseado de forma } \\
\text { inadequada traz riscos ao } \\
\text { meio social e ambiental. }\end{array}$ \\
\hline $\begin{array}{l}\text { Gerenciamento de resíduos } \\
\text { sólidos de saúde em } \\
\text { hospital universitário do } \\
\text { Nordeste } \\
\text { Revista Brasileiro. } \\
\text { Geografia Física. 2019. }\end{array}$ & $\begin{array}{l}\text { Negreiros, R. } \\
\text { V; Araújo, F. } \\
\text { N. F; Silva, } \\
\text { V. F; Souza, } \\
\text { P. M. }\end{array}$ & $\begin{array}{l}\text { Pesquisa quantitativa, } \\
\text { descritiva } \\
\text { transversal realizada } \\
\text { em um hospital em } \\
\text { Campina Grande, } \\
\text { Paraíba. } \\
\text { distribuídos } \\
\begin{array}{l}\text { questionários a } 25 \\
\text { profissionais } \\
\text { enfermagem. }\end{array}\end{array}$ & $\begin{array}{l}\text { Identificou-se a necessidade } \\
\text { da adoção do PGRSS para } \\
\text { um ambiente sustentável, de } \\
\text { modo, torna-se viável } \\
\text { proporcionar educação } \\
\text { continuada para melhor } \\
\text { condição } \\
\text { sustentabilidade, uma vez } \\
\text { que, os resíduos gerados } \\
\text { expõem riscos ao homem e } \\
\text { ao ambiente. }\end{array}$ \\
\hline $\begin{array}{l}\text { Gerenciamento de resíduos } \\
\text { de serviços de saúde em } \\
\text { um hospital no norte do } \\
\text { Rio Grande do Sul. Gestão } \\
\text { e Desenvolvimento em } \\
\text { Revista. } 2020 \text {. }\end{array}$ & $\begin{array}{l}\text { Salomão, A; } \\
\text { Gallon, } \quad \text {; } \\
\text { Mores, G. V. }\end{array}$ & $\begin{array}{l}\text { Uma pesquisa } \\
\text { qualitativa de caráter } \\
\text { exploratória. } \\
\text { Estudo foi realizado } \\
\text { com } 12 \text { profissionais } \\
\text { ligados } \\
\text { gerenciamento de } \\
\text { RSS, entre eles } \\
\text { enfermeiros. }\end{array}$ & 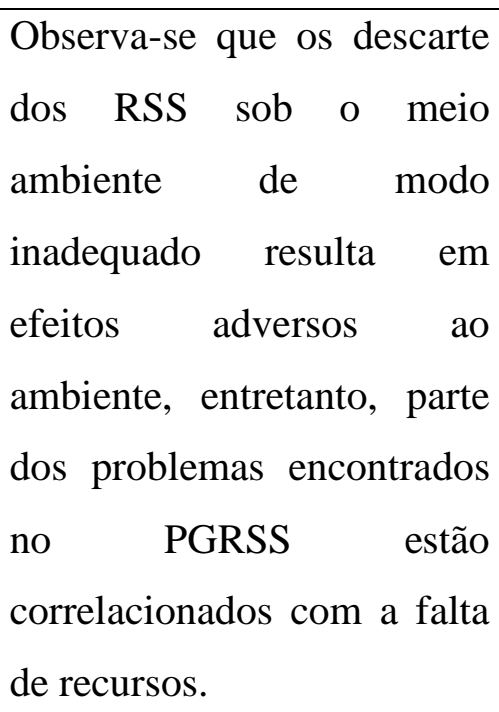 \\
\hline $\begin{array}{l}\text { Percepção de enfermeiros } \\
\text { sobre gerenciamento de } \\
\text { resíduos de serviços de } \\
\text { saúde. Brazilian Journal of } \\
\text { Health Review. } 2020 .\end{array}$ & $\begin{array}{l}\text { Silva, J. T; } \\
\text { Jesus, G. A. } \\
\text { S; Silva, M. } \\
\text { R; Ferreira, S. } \\
\text { M. I. L; Silva, } \\
\text { G. } \quad \text { L; }\end{array}$ & $\begin{array}{l}\text { Estudo descritivo, } \\
\text { exploratório com } \\
\text { abordagem } \\
\text { qualitativa, realizou- } \\
\text { se entrevista com } 11 \\
\text { enfermeiros de } 10\end{array}$ & $\begin{array}{l}\text { Nota-se conhecimento } \\
\text { limitado em relação ao } \\
\text { gerenciamento dos RSS e } \\
\text { seus impactos ao meio } \\
\text { ambiente. É visto a } \\
\text { implementação do PGRSS }\end{array}$ \\
\hline
\end{tabular}




\begin{tabular}{|c|c|c|c|}
\hline & $\begin{array}{l}\text { Monteiro, N. } \\
\text { M. A. T. }\end{array}$ & UBS. & $\begin{array}{l}\text { para a redução dos riscos, } \\
\text { assim como demonstra a } \\
\text { conscientização para a } \\
\text { prática dos } 3 \text { 'Rs' (reduzir, } \\
\text { reutilizar e reciclar). }\end{array}$ \\
\hline $\begin{array}{l}\text { Resíduos dos serviços de } \\
\text { saúde: a responsabilidade } \\
\text { ambiental na visão dos } \\
\text { profissionais da Atenção } \\
\text { Primária. Research, Society } \\
\text { and Development. } 2021 \text {. }\end{array}$ & $\begin{array}{l}\text { Oliveira, L. } \\
\text { L; Souza, P. } \\
\text { M; } \\
\text { Clementino, } \\
\text { F. S; } \\
\text { Almeida, J. L. } \\
\text { S; Cardoso, } \\
\text { L. M. A. }\end{array}$ & 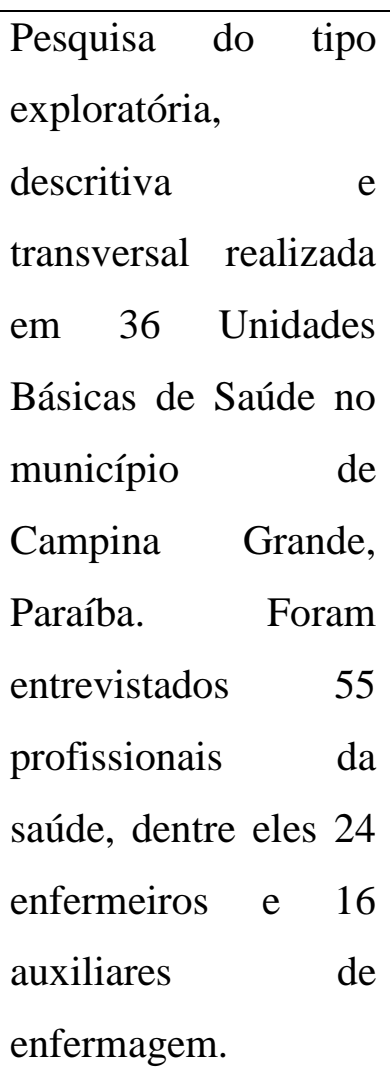 & $\begin{array}{l}\text { É possível notar o } \\
\text { conhecimento sobre a } \\
\text { importância da relação entre } \\
\text { os RSS com o meio } \\
\text { ambiente, bem como um } \\
\text { manejo adequado colabora } \\
\text { para a preservação } \\
\text { ambiental. }\end{array}$ \\
\hline
\end{tabular}

Fonte: Dados da pesquisa, 2021.

\section{DISCUSSÃO}

Aponta-se um conhecimento pouco discutido sobre o conceito de RSS no campo de trabalho em que a enfermagem está inserida. É perceptível que há limitação do saber prático e teórico sobre os resíduos e o seu modo de gerenciamento. Segundo os autores $^{(12)}$, durante o processo de formação, os discentes precisam ter um olhar mais ampliado em relação a produção dos RSS, já que não só se limita a áreas hospitalares, mas abrange, também, estabelecimentos de saúde e o meio ambiente como um todo. Em decorrência disso, a educação voltada pra essa temática deve ter base fortalecida durante o período acadêmico, no que almeja a preservação e minimização dos riscos à saúde ambiental provocados pela prestação do cuidado humano. Desse modo, ao mesmo tempo, evidencia a forma correta de descarte e acondicionamento dos resíduos.

O desconhecimento sobre a forma correta de manusear os resíduos implica na exposição de riscos à saúde, em consequência dos impactos ocasionados ao ambiente. Por esta razão, torna-se fundamental que $o$ https://doi.org/10.31011/reaid-2021-v.95-n.36-art.1255 Rev Enferm Atual In Derme v. 95, n. 36, 2021 e-021182 
gerenciamento dos RSS seja posto de forma consciente a estimular a redução dos riscos de infecções e preservação do meio ambiente ${ }^{(13)}$. É neste ponto onde entra o Plano de Gerenciamento dos Resíduos de Serviços de Saúde (PGRSS), no qual deve corresponder a um conjunto de procedimentos para a gestão correta dos resíduos, com o intuito de promover o compromisso de estabelecer formas preventivas e sustentáveis para o ambiente, firmando a minimização da oferta de impactos a saúde ambiental ${ }^{(14)}$.

Parte da produção dos RSS é consequente das ações da enfermagem, visto que esses profissionais são os que mais geram e manuseiam esses tipos de resíduos durante a assistência. Constatando-se que o manejo seja compreendido de forma adequada desde a segregação até a disposição final, salienta-se que a participação da enfermagem na construção do PGRSS é essencial para o gerenciamento dos resíduos de determinado estabelecimento de saúde. A falta deste, acarreta em danos ambientais pela disposição indiscriminada diante os recursos naturais, uma vez que prejudica a saúde e preservação ambiental. Entretanto, alguns profissionais encontram dificuldades em diferenciar os tipos de resíduos, negligenciando principalmente a etapa da segregação, uma das mais importantes do manejo adequado ${ }^{(15)}$.

Com base nisso, os autores ${ }^{(15)}$, aborda que o enfermeiro tem o poder de gerenciar os serviços de saúde. Além disso, atribui o conhecimento limitado do gerenciamento adequado à sobrecarga de trabalho, falta de discussão na academia e de interesse por parte dos profissionais. Contudo, cerca de 54,16\% dos enfermeiros entrevistados relataram que a etapa de segregação era realizada de maneira adequada, porém $29,16 \%$ destes disseram que não manuseavam de forma correta. Ou seja, a falta de conhecimento sobre o manejo de modo eficiente reflete em efeitos adversos ao ambiente pelo descarte incorreto. Os autores ${ }^{(13)}$, corrobora quando diz que $60,52 \%$ dos profissionais desconhecem a disposição final dos RSS, e ressalta que $92,84 \%$ tem consciência dos riscos inerentes a saúde dos seres vivos.

O descarte incorreto por vezes é negligenciado na fonte geradora, a exemplo da segregação decorrente das falhas no manejo, como a não oferta de recipiente apropriado pra esta destinação, eleva o fator de exposição de riscos aos próprios trabalhadores $^{(16)}$. O mesmo ocorre em decorrência da não apropriação correta e resulta na exposição a periculosidade dos resíduos, visto que se direcionam ao meio ambiente sob a carga de efeitos negativos aos recursos naturais ${ }^{(17)}$.

Outro achado importante em relação ao interesse de cumprir a legislação, é a proposta de pôr em prática nos serviços de saúde a utilização do método 3R's, que se baseia na Redução, Reutilização e Reciclagem dos resíduos, que são viáveis durante o seu 
gerenciamento para a sustentabilidade. Assim, o PGRSS se torna eficiente e sustentável com o meio ${ }^{(18)}$.

A desinformação se apresenta como fator contribuitivo para a ineficácia do manejo adequado, e reforça a perspectiva de proporcionar por meio da educação permanente a oferta de capacitações e treinamentos para o conhecimento acerca do gerenciamento correto, como alternativa para amenizar esses efeitos gerados pela inadequada disposição final dos resíduos sobre o meio natural e ao homem ${ }^{(17)}$.

Visto que o gerenciamento é essencial para manter um ambiente sustentável, bem como propor promoção da saúde, qualidade de vida e a preservação do meio. A associação entre $o$ manejo inadequado com a contaminação do ambiente reflete para a propagação de doenças infectocontagiosas. $\mathrm{Ou}$ seja, quando as substancias provenientes dos resíduos postos sem tratamento e destino adequado pode ultrapassar as barreiras de proteção natural, atingir e contaminar a água de locais superficiais ou subterrâneos, como os lençóis freáticos ${ }^{(3)}$.

Percebe-se que o manejo adequado favorece para a preservação da saúde ambiental e humana, uma vez que contribui para a sustentabilidade. Dessa forma, é através da educação que se conscientiza os profissionais de saúde e por meio das legislações que buscam a iniciativa de programas para a minimização de resíduos e seus riscos ${ }^{(3)}$. Os autores ${ }^{(19)}$ corroboram com a linha de pensamento, quando denotam que as questões ambientais devem ser abordadas nas instituições de saúde e ensino com o intuito de reduzir os impactos ocasionados pelos RSS, e que há necessidade do reconhecer a responsabilidade com o meio social e ambiental.

\section{CONSIDERAÇÕES FINAIS}

Demonstra-se que o conhecimento acerca dos resíduos de serviços de saúde precisa ser discutido pela enfermagem, uma vez que, apresenta fragilidades durante o processo formativo e nas instituições de saúde, no que condiz com a falta de incentivo a práticas de aperfeiçoamento para um bom manejo.

As possíveis consequências provocadas a saúde ambiental e aos homens ocorrem pelo acondicionamento inadequado dos resíduos, visto na contribuição dos profissionais durante as etapas do PGRSS de forma inapropriada, na exposição aos fatores de risco.

Reforça-se, então, a necessidade de aperfeiçoamento da equipe de enfermagem sobre o manejo correto para que se possa colaborar de modo sustentável com o meio ambiente e a saúde. Para tanto, incentiva-se a busca por estudos que complementem esta revisão com o objetivo de alcançar maior 
aprofundamento sobre a temática para futuros pesquisadores.

\section{REFERÊNCIAS}

(1) Brasil. Lei 8.080 de 19/09/1990. Conselho Nacional de Saúde. Brasília, DF. Disponível em:

http://conselho.saude.gov.br/legislacao/lei808 0_190990.htm.

(2) Teixeira JC. Saúde Ambiental. Minas Gerais. Escola de Engenharia Sanitária e Ambiental. Juiz de Fora/Universidade Federal de Juiz de Fora, 2012. Disponível em: https://www.ufjf.br/engsanitariaeambiental/fil es/2012/09/ApostilaSa\%C3\%BAdeAmbiental -E33.pdf.

(3) Oliveira LL, Souza PM, Clementino FS, Almeida JLS, Cardoso NV, Carvalho LMA. Resíduos dos serviços de saúde: a responsabilidade ambiental na visão dos profissionais da Atenção Primária. Research, Society and Development. 2021;10(3): e28610313206-e13206. Disponível em: https://doi.org/10.33448/rsd-v10i3.13206.

(4) Pereira L.R, Nunes JF, Andrade RD. Resíduos de serviços de saúde: uma reflexão sobre seu gerenciamento e os riscos associados. Brazilian Journal of Health Review. 2021;4(3):9988-99. Disponível em: https://www.brazilianjournals.com/index.php/ BJHR/article/view/29337.

(5) Gomes FBM, Silva AKM. Manejo dos resíduos sólidos de serviço em saúde do programa saúde da família- PSF, CaucaiaCeará. Revista SOMMA. 2020;5(2):54-69.

(6) Brasil. Resolução Diretoria Colegiada. Circular $n^{\circ}$ 222, de 28 de março de 2018. Regulamenta Boas Práticas de Gerenciamento de Resíduos. Diário Oficial da União. Publicado no DOU de 29 de março de 2018.

(7) Cavalcante Neto. Desenvolvimento sustentável e meio ambiente: problematizando a geração dos resíduos de saúde. Revista
Saúde e Meio Ambiente. 2019;9(3):23-33. Disponível em: https://trilhasdahistoria.ufms.br/index.php/sa meamb/article/view/8060

(8) Moniz MA, Daher DV, Sabóia VM, Ribeiro CRB. Saúde ambiental: desafios e possibilidades para o cuidado emancipador pelo enfermeiro. Revista Brasileira de Enfermagem. 2020;73. Disponível em: https://doi.org/10.1590/0034-7167-20180478 .

(9) Gutierres ÉD, Rocha LP, Cezar-vaz MR, Yasin JCM, Carvalho DP, Brum RG. Ações de enfermagem com foco no meio ambiente / sustentabilidade voltadas à promoção da saúde das pessoas. Research, Society and Development. 2020; 9(6):e93963556. DOI: 10.33448/rsd-v9i6.3556. Disponível em: https://rsdjournal.org/index.php/rsd/article/vie w/3556.

(10) Sousa LMM, Marques-vieira CMA, Severino SSP, Antunes AV. A metodologia de revisão integrativa da literatura em enfermagem. Revista Investigação em Enfermagem. N²1 Série 2-Novembro 2017, v. 17,2017 . Disponível em: http://www.sinaisvitais.pt/images/stories/Rie/ RIE21.pdf\#page $=17$.

(11) Galvão TF, Pansani TSA, Harrad D. Principais itens para relatar revisões sistemáticas e meta-análises: a recomendação PRISMA. Epidemiologia e Serviços de Saúde [online], Brasília, 2015;24(2). Disponível em: https://www.scielo.br/j/ress/a/TL99XM6YPX 3Z4rxn5WmCNCF/?format=pdf\&lang=pt

(12) Guimarães PSS, Vilela RQB, Oliveira SMB, Silva PJTG, Reis RP, Silva ES, et al. Ensino sobre gerenciamento de resíduos na graduação em enfermagem: realidade, reflexões e propostas. Revista Eletrônica Acervo Saúde. 2021;13(4):e6456-e6456. Disponível em: https://doi.org/10.25248/reas.e6456.2021.

(13) Oliveira LP, Mendonça IVS, Gomes SCS, Caldas AJM. Fatores associados ao 
manejo adequado de resíduos de serviços de saúde entre profissionais de enfermagem. Revista Baiana de Enfermagem. 2018;32. Disponível em: http://dx.doi.org/10.18471/rbe.v32.25104.

(14) Uehara SCSA, Veiga TB, Takayanagui AMM. Gerenciamento de resíduos de serviços de saúde em hospitais de Ribeirão Preto (SP), Brasil. Engenharia Sanitaria e Ambiental. 2019; 24:121-30. Disponível em: https://doi.org/10.1590/S1413-

41522019175893.

(15) Rodrigues AFS, Meireles GOAB, Reis MA, Matos MA, Melo LB, Moura SG, et al. Conhecimento do enfermeiro acerca do gerenciamento dos. Journal of Current Research. 12(4):11167-75. Disponível em: https://www.researchgate.net/profile/Iel-

Filho/publication/343295756_CONHECIME NTO_DO_ENFERMEIRO_ACERCA_DO_ GERENCIAMENTO_DOS_RESIDUOS_DE _SERVICO_DE_SAUDE_NAS_UNIDADES _BASICAS_DE_SAUDE_NO_INTERIOR DE_GOIAS_BRASIL/links/5f21edca299bf13 404925f15/CONHECIMENTO-DO-

ENFERMEIRO-ACERCA-DO-

GERENCIAMENTO-DOS-RESIDUOS-DESERVICO-DE-SAUDE-NAS-UNIDADES-

BASICAS-DE-SAUDE-NO-INTERIOR-DEGOIAS-BRASIL.pdf

(16) Ferreira JPM, Silva MMP, Paiva G. Gerenciamento de Resíduos de Serviços de Saúde em um hospital público em uma grande cidade. Pesquisa, Sociedade e Desenvolvimento. 2020;9(12):e38191211270. Disponível em: https://www.rsdjournal.org/index.php/rsd/arti cle/view/11270

(17) Negreiros RV, Araújo FNF, Silva VF, Souza PM. Gerenciamento de resíduos sólidos de saúde em hospital universitário do Nordeste brasileiro. Revista Brasileira de Geografia Física. 2019;12(1):239-51. Disponível em: https://periodicos.ufpe.br/revistas/rbgfe/article /view/235864.
(18) Silva JT, Jesus GAS, Silva MR, Ferreira SMIL, Silva GL, Monteiro NMAT. Percepção de enfermeiros sobre gerenciamento de resíduos de serviços de saúde. Brazilian Journal of Health Review. 2020;3(6):1636982. Disponível em: https://www.brazilianjournals.com/index.php/ BJHR/article/view/19953.

(19) Salomão A, Gallon S, Mores GV. Gerenciamento de Resíduos de Serviços de Saúde em um Hospital do Norte do Rio Grande do Sul. Gestão e Desenvolvimento em Revista. 2020;6(1):3-14. Disponível em: http://e-

revista.unioeste.br/index.php/gestaoedesenvol vimento/article/view/21806.

\section{Autor correspondente}

Luiz Fernando Brito da Costa, BR 405, KM 153, Arizona 59900-000 - Pau dos FerrosRN. Telefone: +55 (84) 9 9631-4577, e-mail: Luizfernandubrito@gmail.com

Submissão: 2021-10-12

Aprovado: 2021-11-30 